\title{
GRAU DE SATISFAÇÃO DOS PACIENTES EM RELAÇÃO ÀS REFEIÇÕES OFERECIDAS POR UM HOSPITAL PÚBLICO NA CIDADE DE GOIÂNIA - GO
}

Patient satisfaction level in relation to meals offered by a public hospital in the city of Goiânia-GO

Grado de satisfacción de los pacientes en relación con las comidas ofrecidas por un hospital público en la ciudad de Goiânia-GO

\section{Jhessika Ferreira Silva*, Amélia Cristina Stival Duarte, Valéria de Sousa Abreu}

* Correspondência: Diretoria de Ensino e Pesquisa do Hospital Estadual Geral de Goiânia Dr. Alberto Rassi. Avenida Anhanguera $n^{\circ}$ 6.479, Setor Oeste, Goiânia - GO, CEP: 74.110-010. E-mail:

jhessikaferreirasilva@gmail.com.

\section{RESUMO}

O trabalho intitulado de Grau de Satisfação dos Pacientes em Relação às Refeições Oferecidas por um Hospital Público na Cidade de Goiânia-GO, propôs avaliar a existência de correlação de variáveis com a satisfação das refeições. Tratou-se de um estudo transversal, quantitativo, realizado através da aplicação do questionário adaptado de Pfaffenzeller (2003), que abordou as condições sociodemográficas e as características (aparência, temperatura, sabor/tempero, quantidade) avaliadas nas refeições. Amostra foi composta de 100 pacientes com prevalência do

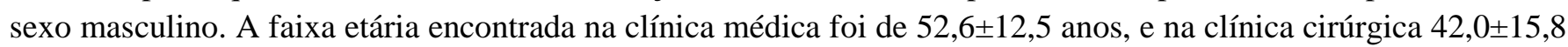
anos. O tempo médio de internação encontrado foi de 7,25 $\pm 6,57$ dias, com diferença significativa $(\mathrm{p}=0,014)$ do tempo de internação entre as clínicas. Com relação a escolaridade, prevaleceu o ensino fundamental incompleto. A renda familiar mensal prevaleceu na faixa de 1 a 3 salários mínimos. Uma prevalência significativa de doenças crônicas na clínica médica e reincidência ao serviço na clínica cirúrgica. Não foi observada diferença entre a consistência e composição da dieta. A maioria dos pacientes relatou estar satisfeito ou muito satisfeito, com diferença estatística somente na aparência $(\mathrm{p}=0,018)$. O presente estudo não encontrou correlação significativa das variáveis analisadas com as características da dieta.

Palavras-chave: Dieta, Internação, Serviço de Alimentação.

\section{ABSTRACT}

The study entitled Degree of Patient Satisfaction in Relation to Meals Offered by a Public Hospital in the City of Goiannia-GO, proposed to evaluate the existence of correlation of variables with meal satisfaction. This was a cross-sectional, quantitative study using the questionnaire adapted from Pfaffenzeller (2003), which addressed the sociodemographic conditions and characteristics (appearance, temperature, flavor / seasoning, quantity) evaluated in meals. Sample was composed of 100 patients with male prevalence. The age group found in the medical clinic was $52.6 \pm 12.5$ years, and in the surgical clinic $42.0 \pm 15.8$ years. The mean time of hospitalization was $7.25 \pm$ 6.57 days, with a significant difference $(p=0.014)$ in the length of hospital stay between the clinics. Regarding schooling, incomplete elementary school prevailed. Monthly family income prevailed in the range of 1 to 3 minimum wages. A significant prevalence of chronic diseases in the medical clinic and recurrence in service in the surgical clinic. There was no difference between the consistency and composition of the diet. The majority of patients reported being pleased or very pleased, with statistical difference only in appearance $(p=0.018)$. The present study did not find a significant correlation of the analyzed variables with the characteristics of the diet.

Keywords: Diet, hospitalization, Food Service. 


\section{RESUMEN}

El trabajo titulado de Grado de Satisfacción de los Pacientes en relación a las Comidas ofrecidas por un Hospital Público en la Ciudad de Goiânia-GO, propuso evaluar la existencia de correlación de variables con la satisfacción de las comidas. Se trató de un estudio transversal, cuantitativo, realizado a través de la aplicación del cuestionario adaptado de Pfaffenzeller (2003), que abordó las condiciones sociodemográficas y las características (apariencia, temperatura, sabor / condimento, cantidad) evaluadas en las comidas. La muestra fue compuesta de 100 pacientes con prevalencia del sexo masculino. El grupo de edad encontrado en la clínica médica fue de 52,6 \pm 12,5 años, y en la clínica quirúrgica 42,0 $\pm 15,8$ años. El tiempo promedio de internación encontrado fue de 7,25 \pm 6,57 días, con diferencia significativa $(p=0,014)$ del tiempo de internación entre las clínicas. Con respecto a la escolaridad, prevaleció la enseñanza fundamental incompleta. La renta familiar mensual prevaleció en el rango de 1 a 3 salarios mínimos. Una prevalencia significativa de enfermedades crónicas en la clínica médica y reincidencia al servicio en la clínica quirúrgica. No se observó diferencia entre la consistencia y la composición de la dieta. La mayoría de los pacientes relató estar satisfecha o muy satisfecha, con diferencia estadística sólo en la apariencia $(p=0,018)$. El presente estudio no encontró correlación significativa de las variables analizadas con las características de la dieta.

Descriptores: Dieta, Internación, Servicio de Alimentación.

\section{INTRODUÇÃO}

De acordo a lei $\mathrm{n}^{\circ} 11.346 / 2006$ todo ser humano tem direito a alimentação adequada independente da etnia cor, credo e cultura. A alimentação é inerente a sobrevivência do homem, ela irá fornecer os nutrientes necessários para funcionamento adequado do seu corpo e permiti-lo executar as tarefas cotidianas (DEMÁRIO et al., 2010). O direito à alimentação, para todos os indivíduos, é garantido constitucionalmente e deve ser assegurando por meio de políticas públicas promotoras de Segurança Alimentar e Nutricional, que visa garantir o acesso regular e constante a alimentos com valores nutricionalmente adequados, em quantidades suficientes e que não comprometa o recebimento de outros bens essenciais (Lei $\left.n^{\circ} 11.346 / 2006\right)$.

Diante desse contexto, destaca-se que a relação do sujeito com o alimento envolve também um sistema de representações, que desloca a ideia do comer da perspectiva biológica para o campo social, cultural e afetivo (CONTRERAS e GRACIA, 2012). Nessa perspectiva, o processo de internação pode causar uma desestruturação na contextualização da DOI: http://dx.doi.org/10.20873/uftv6-6559 relação do sujeito com o alimento por caracterizar um afastamento do cotidiano para outro ambiente, com rotinas e normas rígidas diferentes do seu contexto familiar. Isso pode refletir de forma negativa, levando o paciente a experimentar sentimentos como o medo e a carência (ANTONIO et al., 2002). Por si só, a hospitalização pode ser uma experiência difícil, carregada de aspectos negativos como o distanciamento do ambiente familiar, de pessoas íntimas e, muitas vezes, requerer um certo grau de dependência (ANTONIO et al., 2002).

A alimentação no ambiente hospitalar, por muitos anos, teve uma reputação negativa, os pacientes demonstravam baixa aceitação justificada, na maioria das vezes, pela monotonia das refeições, decorrente da pouca variedade ofertada. A comida estava limitada pelo que a horta do hospital tinha a oferecer (GARCIA, 2006). Além disso, o paciente pode vir a apresentar uma recusa das refeições fornecidas durante a internação por inúmeros fatores, que podem estar relacionados a doença ou a seus sintomas, restrições na composição da dieta, um padrão de comida muito diferente do seu habitual, ou grande volume ofertado, gerando uma baixa aceitação 
e comprometimento do estado nutricional (FERREIRA et al., 2013).

Essa mudança de ambiente por circunstâncias adversas pode conduzir o paciente a transferir a representação hedônica do alimento, interpretando-o como parte do tratamento. Dessa forma, a aceitação das refeições é dada pela associação com uma medicação, necessária à sua recuperação (CORBEU, 2005).

Esse cenário reforça o argumento de que a alimentação não é somente um ato mecânico que visa suprir necessidades nutricionais e biológicas fundamentais à sobrevivência, o ato de realizar uma refeição tem uma dimensão simbólica, subjetiva para o indivíduo (FERREIRA et al., 2013).

$\mathrm{O}$ ato de comer e a comensalidade que ele proporciona têm a capacidade de gerar lembranças, trazer sentimentos, expectativas, remeter ao passado, resgatar na memória episódios alegres ou tristes. A comida é carregada de valores para o indivíduo, o ato de alimentar propicia a uma verdadeira interação de representações e significados que permeiam a sua vida social e afetiva (CORBEU, 2005). Collaço, 2008 evidencia que esses momentos de convivialidade concebidos em torno do alimento são mais frequentes no espaço doméstico, uma vez que a casa permite o resgate afetivo de memórias que favorece a valorização hedônica do alimento. Quando transferido para o contexto hospitalar a interpretação simbólica da refeição é reduzida para o fim terapêutico e o ato de comer passa a ser visto como um procedimento, muitas vezes, passível de recusa (COLLAÇO, 2008).

Contudo as refeições hospitalares devem ser compreendidas a partir de outras interpretações, pois a sequência do comer não se resume ao simples ato que vai da recepção da matéria-prima ao garfo (CORBEU, 2005). A alimentação tem seu papel terapêutico frente a impedir a desnutrição hospitalar que está correlacionada a alta taxa de morbimortalidade e maior tempo de internação (WAITZBERG et al., 1999), mas também existe uma visão mais ampla da importância da alimentação como relevante papel social, em todos os ambientes (CORBEU, 2005).

O objetivo desta pesquisa é identificar o grau de satisfação de pacientes em relação às refeições oferecidas nas clínicas médica e cirúrgica de um hospital público da cidade de Goiânia-GO e identificar se existe relação com o tempo de internação, a patologia de base e outras variáveis associadas com a história pessoal do paciente.

\section{MATERIAIS E MÉTODOS}

O estudo desenvolvido foi de delineamento transversal quantitativo, conduzido nas clínicas médicas e cirúrgica do Hospital Estadual Geral de Goiânia Dr. Alberto Rassi - HGG, Goiânia-GO. Os critérios de inclusão para a seleção da amostra compreenderam: pacientes conscientes que estivessem verbalizando; maiores de 18 anos; de ambos os sexos; internados por no mínimo dois dias; que estivessem com prescrição de dieta de consistências normal, branda, pastosa em todas as suas variações, líquida grossa e as suas variações, que tivessem por limitação da doença restrições de minerais, macronutrientes ou micronutrientes; e que não estivessem em enfermarias de isolamento ou precaução de contato.

A seleção da amostra ocorreu de forma sistemática, e a abordagem dos pacientes procedeu entre os meses de maio a setembro de 2018; Para a realização da pesquisa foi aplicado um questionário adaptado de Pfaffenzeller (2003), que abordou as condições sociodemográficas e questões retrospectivas referentes às características avaliadas 
nas refeições: aparência, temperatura, sabor/tempero e quantidade. Nestes quesitos o questionário possui uma escala que se estende de muito satisfeito, satisfeito, indiferente, pouco satisfeito, insatisfeito. Foram consideradas todas as refeições servidas no hospital: desjejum, colação, almoço, lanche, jantar, ceia e $2^{\circ}$ ceia.

O questionário foi aplicado todos os dias da semana nos pacientes que se encaixaram nos critérios de inclusão. Alguns dados como dieta atual, data da internação, comorbidades, doença de base, motivo da internação, internações pregressas foram coletados por meio de consulta ao prontuário eletrônico do paciente.

As patologias apresentadas pelos participantes foram classificadas como: transtornos cardiovasculares, neoplasias, complicações de diabetes, transtornos gastrointestinais, nefropatia, distúrbio hepatobiliar e outras causas.

Inicialmente, foi realizado um projeto piloto com uma amostra de 10 entrevistados nos períodos matutino e vespertino, com a finalidade de avaliar o questionário e fazer os ajustes pertinentes, identificar dificuldades e estabelecer o método de aplicação.

A aplicação do questionário aconteceu somente uma vez a cada paciente onde ele foi questionado acerca do grau de satisfação das refeições com relação a aparência, temperatura, sabor/tempero, quantidade. Os dados foram coletados após o paciente ser informado sobre os dizeres do Termo de Consentimento Livre e Esclarecido e sua assinatura e a pesquisa foi aprovada pelo Comitê de Ética em Pesquisa do HGG com emissão do Parecer $n^{\circ}$ 201800010018024.

A análise estatística foi realizada no programa IBM SPSS Statistics for Windows 16.0. As variáveis contínuas foram expressas em médias, com seus respectivos desvios padrões; as variáveis categóricas foram apresentadas por meio das frequências absolutas e relativas.

Para comparar as médias e frequências das variáveis em relação à clínica de internação foram utilizados os testes T de Student e teste de MannWhitney, respectivamente. Os possíveis fatores interferentes nas respostas relacionadas a satisfação em relação as refeições foram analisados por meio dos testes de correlação de Spearman. Para todas as análises, foi considerado um nível de significância de $5 \%$ (valor $\mathrm{p}<0,05$ ) e intervalo de confiança de $95 \%$.

\section{RESULTADOS E DISCUSSÃO}

Mediante a apresentação da pesquisa e seus objetivos, foram abordados 111 pacientes, dos quais 7 recusaram participar e 4 foram excluídos por não atenderem os critérios de inclusão do estudo. Dessa forma, a amostra total válida foi composta por 100 pacientes internados nas clínicas médica e cirúrgica.

O tamanho da amostra assemelhou-se ao estudo conduzido por (RIBAS et al., 2013), que investigaram a satisfação das refeições servidas em um hospital público universitário na cidade do Rio de Janeiro conduzido com 132 pacientes e ao estudo realizado por (SANTOS et al., 2012), que avaliaram a aceitação de dietas com restrição de sal por pacientes portadores de cardiopatias em hospital terciário de Porto Alegre RS, conduzido com 175 participantes.

Dos 100 participantes da amostra, 35 foram abordados na clínica médica, sendo 45,7\% mulheres e 54,3\% homens, 65 estavam internados na clínica cirúrgica, sendo 49,2\% mulheres e 50,8\% homens.

Houve uma maior prevalência de homens nas duas clínicas 54,3\%,50,8\% este dado se aproximou do estudo de (RIBAS et al., 2013), em que $56,1 \%$ da amostra foi composta por indivíduos do sexo masculino. Por outro lado (COLOÇO et al., 2009) em 
seu estudo que buscou os determinantes do grau de satisfação das refeições em um hospital universitário da cidade de Campinas São Paulo, encontraram uma maior prevalência de mulheres 52,4\%.

Com relação a diferenciação entre as clínicas das características investigadas, esse mesmo parâmetro foi utilizado por em seu estudo (COLOÇO et al., 2009), onde 80 pacientes na clínica médica e 83 pacientes da clínica cirúrgica.

A faixa etária encontrada na clínica médica foi

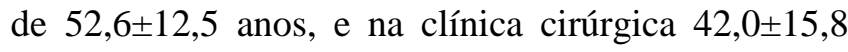
anos. Condiz com o estudo (RIBAS et al., 2013), onde a média de idade foi acima de 45 anos.

O tempo médio de internação encontrado foi de 7,25 $\pm 6,57$ dias, com diferença significativa $(\mathrm{p}=0,014)$ do tempo de internação entre as clínicas, sendo maior o tempo de permanência na clínica

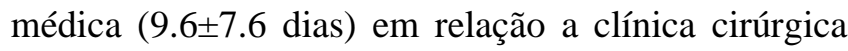
(5.9 \pm 5.5 dias). A recorrência de internação no HGG apresentou uma tendência $(p=0,065)$ em ser mais prevalente entre os pacientes que estavam internados na clínica cirúrgica 44,6\% em relação aos da clínica médica $25,7 \%$. Esses dados não interferiram na aceitação da dieta, resultado que corrobora ao estudo de (COLOÇO et al., 2009), onde não apresentou influência significativa do tempo de internação e reincidência ao hospital. Já o estudo realizado por (RIBAS et al., 2013), 82,6\% dos pacientes que estavam internados há pelo menos duas semanas influenciou de forma negativa na aceitabilidade da dieta, visto que esta decresceu à medida que aumentou o tempo de internação.

Com relação a escolaridade, a maior parte da amostra, em ambas as clínicas, não chegou a concluir o ensino fundamental $34,3 \%$ na médica e $35,4 \%$ na cirúrgica. $\mathrm{Na}$ clínica cirúrgica foi encontrada a mesma prevalência entre ensino fundamental incompleto e ensino médio completo $(35,4 \%$ em ambos). Na pesquisa de (SANTOS et al., 2012), também prevaleceu analfabetos ou aqueles que estudaram até o $4^{\circ}$ ano do ensino fundamental (53\%).

A renda familiar mensal prevaleceu na faixa de 1 a 3 salários mínimos na clínica medica e cirúrgica, $77,1 \%$ e 70,8 respectivamente. Um estudo realizado por (RIBAS et al., 2013), os participantes foram agrupados em classe socioeconômica segundo o Critério de Classificação Econômica do Brasil, e encontraram que pacientes de classe social mais baixa tem mais aceitabilidade que os de maior poder aquisitivo, porém não foi encontrado esta relação na presente pesquisa. Estão demonstrados os dados gerais de saúde entre a população internada nas clínicas médica e cirúrgica, em que é possível observar uma maior prevalência de doenças crônicas nos pacientes abordados na clínica médica (Tabela 1).

Tabela 1. Informações de saúde da amostra investigada

\begin{tabular}{lccccc}
\hline \multirow{2}{*}{ Variável } & \multicolumn{2}{c}{$\begin{array}{c}\text { Clínica Médica } \\
(\mathbf{n}=\mathbf{3 5})\end{array}$} & $\begin{array}{c}\text { Clínica Cirúrgica } \\
(\mathbf{n}=\mathbf{6 5})\end{array}$ & Valor $\mathbf{p}^{*}$ \\
\cline { 2 - 5 } & $\mathbf{n}$ & $\mathbf{\%}$ & $\mathbf{n}$ & $\mathbf{\%}$ & \\
\hline Presença de comorbidades & 27 & 77,1 & 26 & 40,0 & $<0,001$ \\
Diabetes & 11 & 31,4 & 4 & 6,2 & 0,001 \\
Hipertensão Arterial & 19 & 54,3 & 22 & 33,8 & 0,049 \\
Doença Renal Crônica & 4 & 11,4 & 1 & 1,5 & 0,031 \\
Doença cardiovascular & 3 & 8,3 & 2 & 3,1 & 0,232 \\
Internação prévia na unidade & 9 & 25,7 & 29 & 44,6 & 0,065 \\
\hline
\end{tabular}


Dentre os motivos da atual internação os transtornos cardiovasculares foram os mais prevalentes na clínica médica $(\mathrm{p}=0,001)$, seguidos por neoplasia, transtornos gastrointestinais, nefropatia, distúrbios hepatobiliar e outras causas. Na clínica cirúrgica a internação dos pacientes se deu, principalmente, por queixas relacionadas a transtornos gastrointestinais, nefropatia e outras causas, envolvendo os distúrbios do trato urogenital e correções ortopédicas (Tabela 2).

Tabela 2. Dados gerais de saúde da amostra investigada por clínica de internação

\begin{tabular}{lccccc}
\hline \multirow{2}{*}{ Variável } & \multicolumn{2}{c}{$\begin{array}{c}\text { Clínica Médica } \\
(\mathbf{n = 3 5})\end{array}$} & $\begin{array}{c}\text { Clínica Cirúrgica } \\
(\mathbf{n = 6 5 )}\end{array}$ & $\begin{array}{c}\text { Valor } \\
\text { p * }\end{array}$ \\
\cline { 2 - 5 } & $\mathbf{n}$ & $\mathbf{\%}$ & $\mathbf{n}$ & $\mathbf{\%}$ & \\
\hline Motivo da internação atual & & & & & \\
Transtorno cardiovascular & 14 & 40,0 & 0 & 0,0 & 0,001 \\
Neoplasia & 1 & 2,9 & 3 & 4,6 & \\
Complicação de Diabetes & 4 & 11,4 & 0 & 0,0 & \\
Transtorno gastrointestinal & 1 & 2,9 & 16 & 24,6 & \\
Nefropatia & 4 & 11,4 & 16 & 24,6 & \\
Distúrbio hepatobiliar & 2 & 5,7 & 8 & 12,3 & \\
Outras causas & 9 & 25,7 & 22 & 33,8 & \\
\hline
\end{tabular}

* Teste de Mann-Withney

As consistências da dieta no presente estudo, nas duas clínicas houve maior prevalência da consistência normal 74,3\%, 70,8\%. O estudo de (NASCIMENTO et al., 2017) se diferencia deste, pois a consistência mais prevalente foi a branda $61 \%$ seguida de normal $27 \%$ e pastosa $12 \%$.

A maioria dos pacientes relatou estar satisfeita ou muito satisfeita em relação às quatro características investigadas sendo na clínica médica muito satisfeito $48,6 \%$, satisfeito $51,4 \%$, clínica cirúrgica muito satisfeito $26,2 \%$, satisfeito $70,8 \%$, similar ao estudo de (SILVA et al., 2010), em que foi feita uma análise da avaliação da aceitabilidade da dieta hospitalar, foi verificado que $100 \%$ dos pacientes do SUS estavam satisfeitos com o cardápio. Equivalente ao estudo de (NASCIMENTO et al., 2017), onde a dieta hospitalar foi avaliada de boa a ótima por $88,5 \%$ dos participantes internados.
O presente estudo encontrou diferença significativa para a característica aparência entre as clinicas, clínica médica $48,6 \%$ e clínica cirúrgica $26,2 \%,(p=0,0018)$, sendo que os pacientes da clínica médica estavam mais satisfeitos com a aparência. $\mathrm{O}$ estudo de (NASCIMENTO et al., 2017) também analisou este mesmo indicador, mas não encontraram diferença significativa. O estudo de (COLOÇO et al., 2009) apontou diferença significativa com relação ao gênero na avaliação da aparência da dieta, os homens atribuíram menor valor à refeição nesta característica, comportando-se de maneira mais crítica que as mulheres. Um estudo realizado por (ROSSETTO, 2014) avaliou a qualidade do serviço hospitalar e nos paramentos alimentares uma das características investigadas era a aparência da refeição e $72 \%$ dos pacientes da clínica médica responderam ótimo para este quesito. Segundo este autor essa característica é 
um atrativo para a aceitabilidade das refeições, e a sua aceitação irá refletir no tratamento do paciente.

Cada característica avaliada na refeição foi correlacionada com as variáveis idade, tempo de internação, sexo, renda, escolaridade, internação prévia no HGG, presença de comorbidades, consistência da dieta, composição da dieta por meio do coeficiente de correlação de Spearman e não houvera influência nas respostas relacionadas a satisfação pelas refeições $(p>0,05)$.

Dados diferentes foram encontrados na literatura sendo o estudo realizado por (COLOÇO et al., 2009) por meio do qual avaliou-se cada refeição separadamente e por enfermarias. f oi observado que o gênero foi determinante na aceitação da aparência, sabor/tempero e quantidade da dieta e a idade teve influência na avalição do sabor/tempero.

\section{CONCLUSÃO}

O presente estudo não encontrou correlação significativa das variáveis analisadas com as características da dieta, sendo possível inferir que a amostra de número reduzido pode ter influenciado nos resultados.

De modo geral é possível concluir que os pacientes estão satisfeitos com o cardápio alimentar na aparência, sabor/tempero, quantidade e temperatura. Entretanto, sugere-se que mais estudos sejam feitos para análise do estado nutricional e sobre o verdadeiro simbolismo que a dieta tem para esses pacientes.

\section{AGRADECIMENTOS}

Agradecemos aos participantes da pesquisa e ao Hospital que permitiu que este estudo fosse realizado.
Todos os autores declararam não haver qualquer potencial conflito de interesses referente a este artigo.

\section{REFERÊNCIAS}

ANTONIO, P.S, MUNARI, D.B e COSTA, K.H. Fatores geradores de sentimentos do paciente internado frente ao cancelamento de cirurgias. Rev Elet de Enfer. v. 4, n. 1, p. 1518-1944, 2002.

BRASIL. Presidência da República, Subchefia para Assuntos Jurídicos. LEI $\mathrm{N}^{\circ} 11.346$, DE 15 DE SETEMBRO DE 2006. Cria O Sistema Nacional de Segurança Alimentar - SISAN com vista em assegurar o direito humano à alimentação adequada $\mathrm{e}$ dá outras providências. [Internet]. Brasília, DF; 2006. Disponível em

<http://www.planalto.gov.br/ccivil_03/_ato2004-

2006/2006/lei/111346.htm>. Acesso em 2017 obr. 08 obr. 2017.

COLLAÇO, J.H.L. Cozinha doméstica e cozinha profissional: do discurso às práticas. Caderno Espaço Feminino. Uberlâncdia. v.19, n.1, 2008.

COLOÇO, R.B, HOLANDA, L.B e PORTEROMCLELLAN, K.C. Determinantes do grau de satisfação de pacientes internados referente a refeições oferecidas em um hospital universitário. Rev. Ciênc. Méd., Campinas. v. 18, n. 3, p. 121-130, 2009.

CORBEAU, J.P. Alimentar-se no Hospital: as dimensões ocultas da comensalidade. Rev Antropologia e Nutrição: um diálogo possível. 2005.

CONTRERAS. J e GRACIA. M. Alimentação, Sociedade e Cultura. Rev Horiz. Antropol. V.18, n.38, p246, 2012.

DEMÁRIO, R. L.; SOUSA, A. A.; SALES, R. K. Comida de hospital: percepções de pacientes em um hospital público com proposta de atendimento humanizado. Rev. de Ciên. e Saúde Coletiva. V.15, n. $1,2010$.

FERREIRA, D, GUIMARÃES, T. G. e MARCADENTI, A. Aceitação de dietas hospitalares e estado nutricional entre pacientes com câncer. Einstein (São Paulo). v. 11, n. 1, 2013. 
GARCIA, R.V. A dieta hospitalar na perspectiva dos sujeitos envolvidos na sua produção e planejamento. Rev de Nutr. v. 19, n. 2, p. 129-144, 2006.

NASCIMENTO, T, MOREIRA, D.F, CARVALHO, $\mathrm{R}$ de C.R, PEREIRA, M.A.O, PEREIRA, E.A.A, e VILELA, B.S. Aceitabilidade Das Dietas Orais de um Hospital do Sul de Minas. Revista da UIIPS Unidade de Investigação do Instituto Politêcnico de Santarém. v.. 5, n. 5, p. 141-148, 2017.

RIBAS, A.S, PINTO, E de O e RODRIGUES, C.B. Determinantes do grau de aceitabilidade da dieta hospitalar: ferramentas para a prática clínica? Rev Demetra. v. 8, n. 2, p. 137-148, 2013

ROSSETTO, E. Estudo da Qualidade do Serviço Hospitalar do Hospital Universitário Regional dos Campos Gerais por meio do Conceito da Hospitalidade e Humanização. Universidade Estadual do Centro-Oeste. Irati, 2014.
SANTOS, B.F DOS, CAMMERER, M.A e MARCADENTI, A. Aceitação de dietas com reduzido teor de sódio entre cardiopatas em um hospital terciário. Revista Ciência \& Saúde, Porto Alegre, v. 5, n. 2, p. 79-86, 2012.

SILVA, D.O.R, FONSECA, C.S, OLIVEIRA, A.B de, CASTRO, L.C E REZENDE, I de A. Avaliação da Aceitabilidade da Dieta Hospitalar em um Hospital da Cidade de Viçosa, MG. Anais II SIMPAC. v. 2 - n.1, p. 277-282, 2010.

WAITZBERG, D.L, CORREIA, M.I.T.D e CAIAFFA, W.T. Inquérito Brasileiro de Avaliação Nutricional Hospitalar (Ibranutri). Rev Bras Nutr Clin. v. 14, n. 2, p. 124-134, 1999. 